OPEN ACCESS

Edited by:

Weimin Ye,

Karolinska Institutet (KI), Sweden

Reviewed by:

José María Huerta,

Instituto de Salud Carlos III, Spain Francesco Bonomi,

University of Milan, Italy

*Correspondence:

$X i n X u$

drxuxin@zju.edu.cn

Specialty section:

This article was submitted to Nutritional Epidemiology,

a section of the journal

Frontiers in Nutrition

Received: 11 April 2020

Accepted: 27 May 2020

Published: 21 July 2020

Citation:

Xu X (2020) Dairy Product Consumption and Bladder Cancer Risk in the Prostate, Lung, Colorectal, and Ovarian (PLCO) Cohort.

Front. Nutr. 7:97.

doi: 10.3389/fnut.2020.00097

\section{Dairy Product Consumption and Bladder Cancer Risk in the Prostate, Lung, Colorectal, and Ovarian (PLCO) Cohort}

$X i n X u^{*}$

Department of Urology, School of Medicine, The First Affiliated Hospital, Zhejiang University, Hangzhou, China

Evidence has suggested that dairy product consumption lowers the risk of several cancers, but these benefits may not occur with bladder cancer. In a cohort of 101,721 subjects in the Prostate, Lung, Colorectal, and Ovarian (PLCO) Cancer Screening Trial, we analyzed the effects of dairy product intake on bladder cancer risk using Cox proportional hazards regression. After a median of 12.5 years of follow-up, 776 new cases of bladder cancer were identified. We found no statistically significant association between total milk intake and bladder cancer risk. The multivariate-adjusted hazard ratio $(\mathrm{HR})$ of bladder cancer for participants in the highest category of total milk intake compared with those in the lowest category was 1.13 (95\% Cl: 0.92-1.40; $p$ for trend $=0.436$ ). Among individual dairy foods, no statistically significant association was observed for a broad range of dairy products, including whole milk, 2\% milk, 1\% milk, skim milk, yogurt, regular butter, low fat butter, regular cheese, low fat cheese, and no fat cheese. These associations were not modified by smoking status ( $p$ for interaction $>0.05)$. In conclusion, findings from this large prospective analysis do not support an inverse association between dairy product consumption and bladder cancer risk.

Keywords: dairy product, bladder cancer, PLCO, cohort, risk

\section{INTRODUCTION}

Bladder cancer is the 10th most common cancer worldwide, with an estimated 549,000 new cases and 200,000 deaths in $2018(1,2)$. The most well-established risk factors for bladder cancer are cigarette smoking and occupational exposure to aromatic amines and 4,4'-methylenebis (2chloroaniline) (3). Less-established risk factors for bladder cancer include lack of physical activity (4), obesity (5), a history of urinary calculi (6), and chronic urinary tract infection (7).

Recently, several epidemiological studies have shown a potential inverse association between intake of dairy products and bladder cancer risk and a meta-analysis reported that yogurt consumption was significantly associated with a decreased risk of bladder cancer (8). However, the two prospective studies $(9,10)$ included in this meta-analysis have reported inconsistent results. Another meta-analysis by Bermejo et al. (11) also indicated a reduced risk of bladder cancer associated with medium consumption of total dairy products and with medium and high consumption of milk and fermented dairy products. However, a 
pooled analysis of 13 cohort studies by Acham et al. (12) failed to find a significant association between total or individual dairy products and bladder cancer risk. This study aims at contributing to this debate by considering the association between dairy product consumption and bladder cancer risk in the prostate, lung, colorectal, and ovarian (PLCO) cohort.

\section{MATERIALS AND METHODS}

\section{Data Sources}

The PLCO study is a large population-based cancer screening trial designed to evaluate whether selected screening methods could reduce mortality from prostate, lung, colorectal, and ovarian cancer, as described previously (13). Briefly, 154,952 individuals aged $55-74$ years were recruited via 10 centers in the United States between 1993 and 2001. Subjects were included in this study if they completed the baseline questionnaire and were cancer free before completion of the diet history questionnaire (DHQ). All participants provided written informed consent, and the study was approved by the Institutional Review Boards at the National Cancer Institute.

\section{Data Collection}

The baseline questionnaire included self-reported information on demographics (e.g., age, sex, ethnicity, and education), smoking status, family history of cancer, and medical history. Dietary data were collected using the DHQ (14), which included the portion size and frequency of intake of 124 food items and supplement use during the past year. The amount of dairy product intake was calculated using the detailed analysis file output by DietCalc, which determines the gram amounts by sex and serving size using a nutrient database based on national dietary data (USDA's 1994-96 Continuing Survey of Food Intakes by Individuals, available from the USDA Food Surveys Research Group) (15).

\section{Ascertainment of Bladder Cancer}

Study participants were mailed a questionnaire annually to screen cancer cases. All reports of bladder cancer were followed up and medical records were abstracted and reviewed for case ascertainment. In this analysis, bladder cancer case was defined according to International Classification of Diseases for Oncology Second Edition (ICD-O-2), codes C67.0-C67.9. Vital status was obtained by the administration of the Annual Study Update questionnaires, reports from relatives, friends, or physicians, and National Death Index searches.

\section{Statistical Analysis}

Cox proportional hazards regression was used to estimate hazard ratios (HRs) and 95\% confidence intervals (CIs). Models were adjusted for age (categorical), sex (male vs. female), race (White, Non-Hispanic vs. Other), body mass index at the time of enrollment ( $<25$ vs. $\geq 25 \mathrm{~kg} / \mathrm{m}^{2}$ ), education ( $\leq$ high school vs. $\geq$ some college), smoking status (never vs. former $\leq 15$ years since quit vs. former $>15$ years since quit vs. former year since quit unknown vs. current smoker $\leq 1$ pack per day vs. current smoker $>1$ pack per day vs. current smoker intensity unknown), vegetable intake (continuous), fruit intake (continuous), tea intake (continuous), alcohol drinking status (never vs. former vs. current), total energy intake (continuous), randomization arm (intervention vs. control), family history of any cancer (yes vs.

\begin{tabular}{|c|c|c|c|}
\hline Variables & $\begin{array}{c}\text { Non-cases } \\
(n=100,945)\end{array}$ & $\begin{array}{c}\text { Cases } \\
(n=776)\end{array}$ & $p$-value \\
\hline Age (years), mean $\pm S D$ & $62.4 \pm 5.3$ & $64.1 \pm 5.2$ & $<0.001$ \\
\hline \multicolumn{4}{|l|}{$\operatorname{Sex}(n, \%)$} \\
\hline Male & $48,847(48.4)$ & 627 (80.8) & \multirow[t]{2}{*}{$<0.001$} \\
\hline Female & 52,098 (51.6) & $149(19.2)$ & \\
\hline \multicolumn{4}{|l|}{ Smoking status $(n, \%)$} \\
\hline Never & 48,363 (47.9) & $189(24.4)$ & \multirow[t]{4}{*}{$<0.001$} \\
\hline Current & $9264(9.2)$ & $131(16.9)$ & \\
\hline Former & $43,305(42.9)$ & $456(58.8)$ & \\
\hline Missing & $13(0.0)$ & $0(0.0)$ & \\
\hline \multicolumn{4}{|l|}{ Education ( $n, \%)$} \\
\hline$\leq$ High school & $42,574(42.2)$ & $354(45.6)$ & \multirow[t]{3}{*}{0.066} \\
\hline$\geq$ Some college & $58,177(57.6)$ & $419(54.0)$ & \\
\hline Missing & $194(0.2)$ & $3(0.4)$ & \\
\hline \multicolumn{4}{|l|}{ BMI $(n, \%)$} \\
\hline$<25.0 \mathrm{~kg} / \mathrm{m}^{2}$ & 33,522 (33.2) & 217 (28.0) & \multirow[t]{3}{*}{0.008} \\
\hline$\geq 25.0 \mathrm{~kg} / \mathrm{m}^{2}$ & $66,099(65.5)$ & $549(70.7)$ & \\
\hline Missing & $1324(1.3)$ & $10(1.3)$ & \\
\hline \multicolumn{4}{|l|}{$\operatorname{Race}(n, \%)$} \\
\hline White, Non-Hispanic & 91,765 (90.9) & 738 (95.1) & \multirow[t]{3}{*}{$<0.001$} \\
\hline Other & $9143(9.1)$ & $38(4.9)$ & \\
\hline Missing & $37(0.0)$ & $0(0.0)$ & \\
\hline \multicolumn{4}{|l|}{$\begin{array}{l}\text { Alcohol drinking status } \\
(n, \%)\end{array}$} \\
\hline Never & $10,061(10.0)$ & $52(6.7)$ & \multirow[t]{4}{*}{0.003} \\
\hline Former & $14,650(14.5)$ & $105(13.5)$ & \\
\hline Current & $73,368(72.7)$ & $604(77.8)$ & \\
\hline Missing & $2866(2.8)$ & $15(1.9)$ & \\
\hline $\begin{array}{l}\text { Total energy (kcal/day), } \\
\text { median (IQR) }\end{array}$ & $\begin{array}{c}1608 \\
(1222-2102)\end{array}$ & $\begin{array}{c}1742 \\
(1306-2255)\end{array}$ & $<0.001$ \\
\hline \multicolumn{4}{|l|}{$\begin{array}{l}\text { Randomization arm } \\
(n, \%)\end{array}$} \\
\hline Intervention & $51,440(51.0)$ & $364(46.9)$ & \multirow[t]{2}{*}{0.025} \\
\hline Control & 49,505 (49.0) & $412(53.1)$ & \\
\hline \multicolumn{4}{|l|}{ Marital status (n, \%) } \\
\hline Married & 78,972 (78.2) & 639 (82.3) & \multirow[t]{3}{*}{0.006} \\
\hline Not married & $21,790(21.6)$ & $134(17.3)$ & \\
\hline Missing & $183(0.2)$ & $3(0.4)$ & \\
\hline \multicolumn{4}{|l|}{$\begin{array}{l}\text { Family history of any } \\
\text { cancer }(n, \%)\end{array}$} \\
\hline Yes & $56,388(56.0)$ & $450(58.1)$ & \multirow[t]{2}{*}{0.236} \\
\hline No & 44,557 (44.0) & $326(41.9)$ & \\
\hline
\end{tabular}

PLCO, prostate, lung, colorectal and ovarian; SD, standard deviation; BMI, body mass index; IQR, interquartile range. 
TABLE 2 | Association between intake of dairy products and bladder cancer risk in the PLCO cancer screening trial.

\begin{tabular}{|c|c|c|c|c|c|}
\hline Variables (g/day) & Median (g/day) & Cohort (n) & Cases (n) & $\begin{array}{l}\text { Age- and sex-adjusted } \\
\text { HR }(95 \% \mathrm{Cl}), p \text {-value }\end{array}$ & $\begin{array}{l}\text { Multivariable adjusted } \\
\text { HR }(95 \% \mathrm{Cl})^{*}, p \text {-value }\end{array}$ \\
\hline
\end{tabular}

Total milk

Q1 $(<30.02)$

Q2 $(\geq 30.02$ to $<115.88)$

Q3 ( $\geq 115.88$ to $<285.49)$

Q4 ( $\geq 285.49)$

Whole milk"

Q1 (0)

Q2 (>0 to $<21.47)$

Q3 ( $\geq 21.47$ to $<122.22)$

Q4 ( $\geq 122.22)$

2\% milk ${ }^{\#}$

Q1 (0)

Q2 (>0 to <40.39)

Q3 ( $\geq 40.39$ to $<161.04)$

Q4 ( $\geq 161.04)$

$1 \%$ milk ${ }^{\#}$

Q1 (0)

Q2 (>0 to <67.01)

Q3 ( $\geq 67.01$ to $<223.74)$

Q4 ( $\geq 223.74)$

Skim milk\#

Q1 (0)

Q2 (>0 to <77.63)

Q3 ( $\geq 77.63$ to $<261.65)$

Q4 ( $\geq 261.65)$

Yogurt ${ }^{\#}$

Q1 (0)

Q2 (>0 to $<5.42)$

Q3 $(\geq 5.42$ to $<19.04)$

Q4 ( $\geq 19.04)$

Regular butter\#

Q1 (0)

Q2 (>0 to $<0.82)$

Q3 $(\geq 0.82$ to $<3.76)$

Q4 ( $\geq 3.76)$

Low-fat butter\#

Q1 (0)

Q2 (>0 to $<0.37$ )

Q3 $(\geq 0.37$ to $<1.49)$

Q4 ( $\geq 1.49)$

0.00

0.25

1.88

7.75

0.00

0.13

0.76

3.31

85,311

5577

5377

5456

25,808

26,914

161

207

184

224

688

16

36

36

510

84

82

100

0,490

84,667

5685

5725

5644

59,591

14,051

14,044

14,035

41,006

23,586

18,860

18,269

35,545

22,150

21,990

22,036

Regular cheese

Q1 (<0.09)

0.00

0.74
HR (95\% Cl), p-value

HR $(95 \% \mathrm{Cl})^{\star}$, $p$-value

$1.15(0.93-1.41), p=0.193$

$0.97(0.78-1.19), p=0.750$

$1.10(0.90-1.35), p=0.346$

$p$ for trend $=0.590$

Reference group

0.59 (0.36-0.97), $p=0.036$

$1.25(0.89-1.75), p=0.192$

$1.09(0.78-1.52), p=0.627$

$p$ for trend $=0.462$

Reference group

$1.18(0.93-1.48), p=0.170$

$1.02(0.81-1.29), p=0.853$

$1.10(0.89-1.36), p=0.392$

$p$ for trend $=0.484$

Reference group

$0.76(0.53-1.09), p=0.135$

$0.84(0.62-1.16), p=0.292$

$1.06(0.80-1.40), p=0.688$

$p$ for trend $=0.873$

Reference group

$1.08(0.87-1.34), p=0.468$

$0.85(0.68-1.07), p=0.164$

$0.96(0.78-1.19), p=0.713$

$p$ for trend $=0.522$

Reference group

0.83 (0.68-1.00), $p=0.053$

$0.84(0.69-1.03), p=0.096$

$0.78(0.62-0.99), p=0.038$

$p$ for trend $=0.095$

Reference group

$0.95(0.79-1.16), p=0.625$

$1.02(0.84-1.23), p=0.833$

$0.93(0.77-1.14), p=0.499$

$p$ for trend $=0.570$

Reference group

$0.76(0.54-1.08), p=0.126$

$0.93(0.68-1.26), p=0.624$

$0.78(0.56-1.08), p=0.135$

$p$ for trend $=0.134$

Reference group

$1.11(0.91-1.37), p=0.303$
Reference group

$1.16(0.94-1.42), p=0.171$

$0.99(0.80-1.22), p=0.910$

$1.13(0.92-1.40), p=0.243$

$p$ for trend $=0.436$

Reference group

$0.58(0.35-0.95), p=0.031$

$1.13(0.81-1.60), p=0.470$

0.97 (0.69-1.36), $p=0.860$

$p$ for trend $=0.990$

Reference group

$1.11(0.88-1.40), p=0.369$

$0.99(0.78-1.25), p=0.919$

1.03 (0.83-1.29), $p=0.779$

$p$ for trend $=0.880$

Reference group

0.77 (0.54-1.10), $p=0.147$

0.87 (0.63-1.19), $p=0.390$

$1.10(0.83-1.46), p=0.500$

$p$ for trend $=0.641$

Reference group

$1.14(0.92-1.42), p=0.231$

$0.92(0.73-1.15), p=0.449$

1.05 (0.85-1.30), $p=0.664$

$p$ for trend $=0.866$

Reference group

$0.94(0.77-1.14), p=0.515$

$1.00(0.81-1.22), p=0.968$

$0.93(0.73-1.19), p=0.560$

$p$ for trend $=0.640$

Reference group $0.99(0.81-1.20), p=0.895$ $1.01(0.84-1.23), p=0.881$ $0.88(0.72-1.08), p=0.213$

$p$ for trend $=0.191$

Reference group $0.83(0.59-1.18), p=0.303$ $0.98(0.72-1.34), p=0.893$ $0.80(0.57-1.11), p=0.180$

$p$ for trend $=0.190$

Reference group

$1.08(0.88-1.33), p=0.454$

(Continued) 
TABLE 2 | Continued

\begin{tabular}{|c|c|c|c|c|c|}
\hline Variables (g/day) & Median (g/day) & Cohort (n) & Cases $(n)$ & $\begin{array}{l}\text { Age- and sex-adjusted } \\
\text { HR }(95 \% \mathrm{Cl}), p \text {-value }\end{array}$ & $\begin{array}{l}\text { Multivariable adjusted } \\
\text { HR }(95 \% \mathrm{Cl})^{\star}, p \text {-value }\end{array}$ \\
\hline Q3 $(\geq 1.85$ to $<7.38)$ & 3.85 & 24,892 & 194 & $1.09(0.89-1.34), p=0.409$ & $1.02(0.83-1.25), p=0.874$ \\
\hline Q4 ( $\geq 7.38)$ & 13.78 & 24,107 & 222 & $\begin{array}{c}1.19(0.98-1.45), p=0.087 \\
p \text { for trend }=0.147\end{array}$ & $\begin{array}{c}1.11(0.90-1.37), p=0.336 \\
p \text { for trend }=0.424\end{array}$ \\
\hline \multicolumn{6}{|l|}{ Low-fat cheese ${ }^{\#}$} \\
\hline Q1 (0) & 0.00 & 55,534 & 461 & Reference group & Reference group \\
\hline Q2 (>0 to <0.89) & 0.28 & 15,589 & 125 & $1.03(0.84-1.25), p=0.803$ & $1.12(0.91-1.37), p=0.291$ \\
\hline Q3 ( $\geq 0.89$ to $<3.07)$ & 1.83 & 15,354 & 87 & $0.75(0.59-0.94), p=0.012$ & $0.78(0.62-0.98), p=0.036$ \\
\hline Q4 ( $\geq 3.07)$ & 6.08 & 15,244 & 103 & $\begin{array}{c}0.84(0.68-1.04), p=0.113 \\
p \text { for trend }=0.051\end{array}$ & $\begin{array}{c}0.88(0.71-1.09), p=0.244 \\
p \text { for trend }=0.109\end{array}$ \\
\hline \multicolumn{6}{|l|}{ No-fat cheese $\#$} \\
\hline Q1 (0) & 0.00 & 76,813 & 603 & Reference group & Reference group \\
\hline Q2 (>0 to <0.47) & 0.19 & 8688 & 58 & $0.90(0.68-1.17), p=0.430$ & $0.96(0.73-1.27), p=0.790$ \\
\hline Q3 $(\geq 0.47$ to $<2.00)$ & 1.01 & 8156 & 60 & $0.88(0.68-1.15), p=0.348$ & $0.91(0.70-1.19), p=0.489$ \\
\hline Q4 ( $\geq 2.00)$ & 4.53 & 8064 & 55 & $\begin{array}{c}0.84(0.64-1.11), p=0.216 \\
p \text { for trend }=0.194\end{array}$ & $\begin{array}{c}0.85(0.64-1.12), p=0.252 \\
p \text { for trend }=0.229\end{array}$ \\
\hline
\end{tabular}

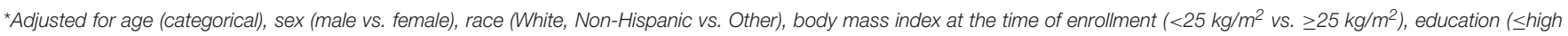

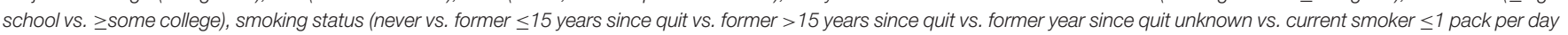

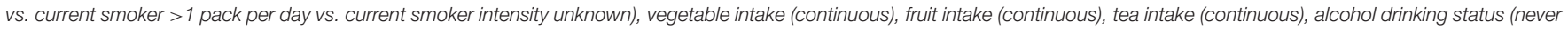

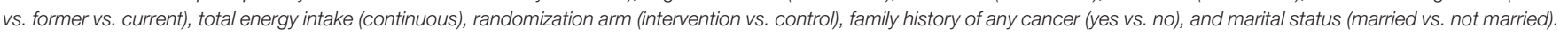
\#Dairy products were categorized using non-consumers as the first category and tertiles of distribution for dairy products consumers.

no), and marital status (married vs. not married). Missing values for covariates were treated as dummy variables in the models. $p$ for trend was calculated using a continuous variable created from medians within quartiles of dietary products. Likelihood ratio tests were used to test violations of the Cox proportional hazards assumption and heterogeneity of associations. A restricted cubic spline model (16) with three knots (i.e., 10th, 50th, and 90th percentiles) was also used to examine the association between dietary total milk intake and bladder cancer risk. All statistical analyses were performed using the software STATA version 15 (Stata Corp, College Station, TX, USA). All tests were two-sided.

\section{RESULTS}

After a median of 12.5 years of follow-up, 776 new cases of bladder cancer were identified from the 101,721 individuals included in our study. Cases were older, were more often men, and were more likely to be current smokers or ex-smokers. Table 1 shows the main characteristics of included subjects with and without bladder cancer.

We found no statistically significant association between total milk intake and risk of bladder cancer (Table 2). The multivariate-adjusted HR of bladder cancer for participants in the highest category of total milk intake ( $525.87 \mathrm{~g} /$ day) compared with those in the lowest category (5.49 g/day) was 1.13 (95\% CI: $0.92-1.40 ; p$ for trend $=0.243$ ). A spline regression plot of bladder cancer risk in relation to total milk intake is shown in Figure 1. There was no statistical evidence for non-linearity ( $p$ for non-linearity $>0.05$ ). When stratifying by sex, the results were not substantially different. Total milk intake was not associated with bladder cancer risk either in male $\left(\mathrm{HR}_{\mathrm{Q} 4 \mathrm{vs} Q 1}\right.$

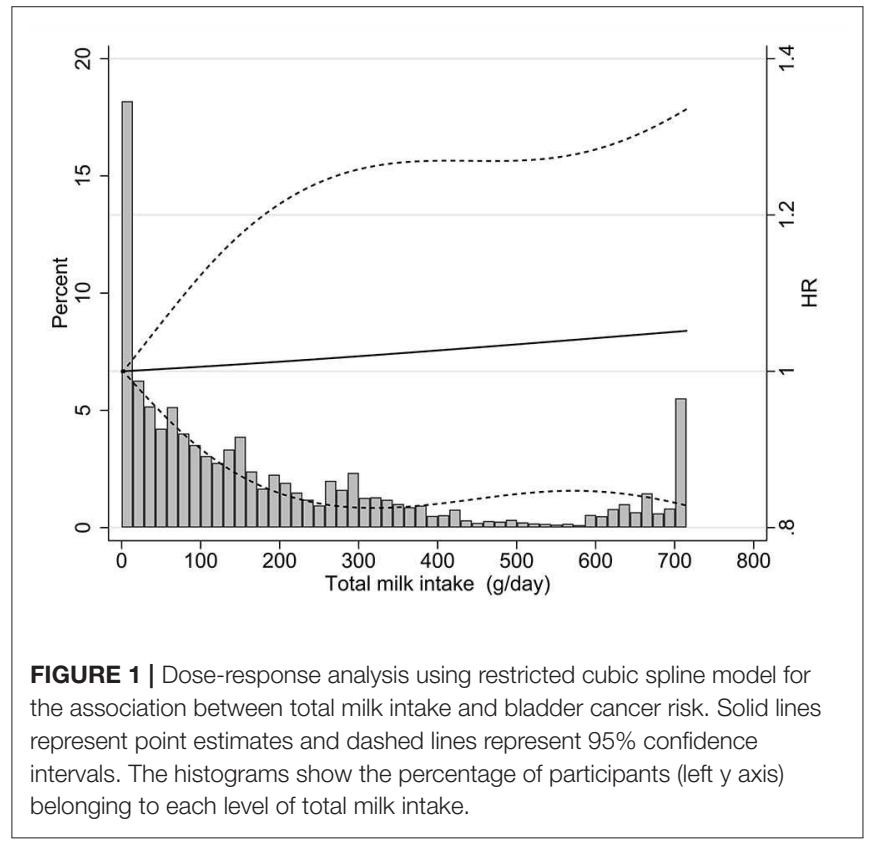

$=1.06,95 \% \mathrm{CI}=0.84-1.34)$ or in female $\left(\mathrm{HR}_{\mathrm{Q} 4 \mathrm{vs} \mathrm{Q}}=1.26\right.$, 95\% CI $=0.80-1.98)$.

Among individual dairy foods, no statistically significant association was observed for a broad range of dairy products, including whole milk, $2 \%$ milk, $1 \%$ milk, skim milk, yogurt, regular butter, low-fat butter, regular cheese, low-fat cheese, and no-fat cheese (Table 2). These associations were not modified by smoking status ( $p$ for interaction $>0.05$ ). 


\section{DISCUSSION}

In this large prospective study, various non-fermented and fermented dairy products were not associated with bladder cancer risk, in age- and sex-adjusted models or in multivariableadjusted models. The null associations did not differ among strata defined by smoking status.

An updated WCRF-AICR Continuous Update Project in 2017 indicated that colorectal cancer risk decreased by $13 \%$ for each $400 \mathrm{~g} /$ day increment of dairy product consumption (95\% CI: 10$17 \%)$ (17). Similarly, four recent meta-analyses $(8,11,18,19)$ reported an inverse association between intake of dairy products, especially fermented dairy foods and bladder cancer risk. These associations may differ by geographical region. For example, the meta-analysis by Mao et al. (19) reported a significant protective effect of milk consumption on bladder cancer in Asia but not in Europe. However, these meta-analysis included both casecontrol and cohort studies. Evidence from prospective studies was relatively limited and inconsistent $(9,10)$. Keszei et al. (9) reported that total dairy intake was not associated with bladder cancer risk in the Netherlands Cohort Study on Diet and Cancer, although there was some weak evidence that bladder cancer risk was inversely associated with fermented dairy products. Larsson et al. (10) also found no association between total dairy intake and bladder cancer risk in a prospective study of Swedish women and men. However, their study provided some evidence that a high intake of cultured milk may lower the risk of bladder cancer. Therefore, we undertook this analysis in PLCO study, and as a result, we found no evidence that intake of raw or fermented dairy was associated with the risk of bladder cancer.

Strengths of the PLCO study included the prospective design, large sample size, and high completeness of follow-up, which minimized the selection bias. Additionally, the information of main potential confounders for bladder cancer was available. Finally, we could analyze non-fermented and fermented milk separately and further perform analyses on dairy products with different fat contents. The limitations of PLCO included no repeated measurement of dietary product consumption during

\section{REFERENCES}

1. Bray F, Ferlay J, Soerjomataram I, Siegel RL, Torre LA, Jemal A. Global cancer statistics 2018: GLOBOCAN estimates of incidence and mortality worldwide for 36 cancers in 185 countries. CA Cancer J Clin. (2018) 68:394424. doi: $10.3322 /$ caac. 21492

2. Ferlay J, Colombet M, Soerjomataram I, Mathers C, Parkin DM, Pineros $\mathrm{M}$, et al. Estimating the global cancer incidence and mortality in 2018: GLOBOCAN sources and methods. Int J Cancer. (2019) 144:194153. doi: 10.1002/ijc.31937

3. Letasiova S, Medve'ova A, Sovcikova A, Dusinska M, Volkovova K, Mosoiu $\mathrm{C}$, et al. Bladder cancer, a review of the environmental risk factors. Environ Health. (2012) 11(Suppl 1):S11. doi: 10.1186/1476-069X-11-S1-S11

4. Keimling M, Behrens G, Schmid D, Jochem C, Leitzmann MF. The association between physical activity and bladder cancer: systematic review and metaanalysis. Br J Cancer. (2014) 110:1862-70. doi: 10.1038/bjc.2014.77

5. Qin Q, Xu X, Wang X, Zheng XY. Obesity and risk of bladder cancer: a meta-analysis of cohort studies. Asian Pac J Cancer Prev. (2013) 14:311721. doi: 10.7314/APJCP.2013.14.5.3117 follow-up (the amount of dietary product consumption may change during follow-up), potential misclassification with selfreported questionnaire, and possible residual confounding (e.g., physical activity).

In summary, this study did not support the hypothesis that intake of dairy products was associated with the risk of bladder cancer. Further well-designed large prospective studies or collaborative studies are still warranted to verify our findings.

\section{DATA AVAILABILITY STATEMENT}

The datasets presented in this article are not readily available because the data that support the findings of this study are available from NIH PLCO study group. Restrictions apply to the availability of these data, which were used under license for this study. Requests to access the datasets should be directed to https://biometry.nci.nih.gov/cdas/datasets/plco/.

\section{ETHICS STATEMENT}

The studies involving human participants were reviewed and approved by the Institutional Review Boards at the National Cancer Institute. The patients/participants provided their written informed consent to participate in this study.

\section{AUTHOR CONTRIBUTIONS}

XX designed the study, performed the data analyses, and drafted the manuscript. All authors contributed to the article and approved the submitted version.

\section{ACKNOWLEDGMENTS}

The author thanks the National Cancer Institute for access to NCI's data collected by the Prostate, Lung, Colorectal, and Ovarian (PLCO) Cancer Screening Trial. The statements contained herein are solely those of the author and do not represent or imply concurrence or endorsement by NCI.
6. Yu Z, Yue W, Jiuzhi L, Youtao J, Guofei Z, Wenbin G. The risk of bladder cancer in patients with urinary calculi: a meta-analysis. Urolithiasis. (2018) 46:573-9. doi: 10.1007/s00240-017-1033-7

7. Akhtar S, Al-Shammari A, Al-Abkal J. Chronic urinary tract infection and bladder carcinoma risk: a meta-analysis of case-control and cohort studies. World J Urol. (2018) 36:839-48. doi: 10.1007/s00345-018-2206-x

8. Zhang K, Dai H, Liang W, Zhang L, Deng Z. Fermented dairy foods intake and risk of cancer. Int J Cancer. (2019) 144:2099-108. doi: 10.1002/ijc.31959

9. Keszei AP, Schouten LJ, Goldbohm RA, van den Brandt PA. Dairy intake and the risk of bladder cancer in the Netherlands cohort study on diet and cancer. Am J Epidemiol. (2010) 171:436-46. doi: 10.1093/aje/kwp399

10. Larsson SC, Andersson SO, Johansson JE, Wolk A. Cultured milk, yogurt, and dairy intake in relation to bladder cancer risk in a prospective study of Swedish women and men. Am J Clin Nutr. (2008) 88:10837. doi: 10.1093/ajcn/88.4.1083

11. Bermejo LM, López-Plaza B, Santurino C, Cavero-Redondo I, GómezCandela C. Milk and dairy product consumption and bladder cancer risk: a systematic review and meta-analysis of observational studies. Adv Nutr. (2019) 10(suppl 2):S224-S38. doi: 10.1093/advances/nmy119 
12. Acham M, Wesselius A, van Osch FHM, Yu EY-W, van den Brandt PA, White E, et al. Intake of milk and other dairy products and the risk of bladder cancer: a pooled analysis of 13 cohort studies. Eur J Clin Nutr. (2020) 74:28-35. doi: 10.1038/s41430-019-0453-6

13. Prorok PC, Andriole GL, Bresalier RS, Buys SS, Chia D, Crawford $\mathrm{ED}$, et al. Design of the prostate, lung, colorectal and ovarian (PLCO) cancer screening trial. Control Clin Trials. (2000) $21(6$ Suppl):273S-309S. doi: 10.1016/S0197-2456(00)00098-2

14. Diet history questionnaire. Risk Factor Monitoring and Methods. National Cancer Institute. Version current 30 Nov 2016. Available online at: http:// www.riskfactor.cancer.gov/DHQ (accessed Novomber 30, 2016).

15. Cancer Data Access System. DQX Dataset: Data Dictionary, Appendix 2: Nutrient. Washington, DC: US Department of Health and Human Services (2013).

16. Marrie RA, Dawson NV, Garland A. Quantile regression and restricted cubic splines are useful for exploring relationships between continuous variables. J Clin Epidemiol. (2009) 62:511-7.e1. doi: 10.1016/j.jclinepi.2008. 05.015

17. Vieira AR, Abar L, Chan DSM, Vingeliene S, Polemiti E, Stevens C, et al. Foods and beverages and colorectal cancer risk: a systematic review and meta-analysis of cohort studies, an update of the evidence of the WCRF-AICR Continuous update project. Ann Oncol. (2017) 28:1788802. doi: $10.1093 /$ annonc/mdx171

18. Wu J, Yu Y, Huang L, Li Z, Guo P, Xu YW. Dairy product consumption and bladder cancer risk: a meta-analysis. Nutr Cancer. (2019) 72:377385. doi: 10.1080/01635581.2019.1637909

19. Mao QQ, Dai Y, Lin YW, Qin J, Xie LP, Zheng XY. Milk consumption and bladder cancer risk: a meta-analysis of published epidemiological studies. Nutr Cancer. (2011) 63:1263-71. doi: 10.1080/01635581.2011.614716

Conflict of Interest: The author declares that the research was conducted in the absence of any commercial or financial relationships that could be construed as a potential conflict of interest.

Copyright $\odot 2020 \mathrm{Xu}$. This is an open-access article distributed under the terms of the Creative Commons Attribution License (CC BY). The use, distribution or reproduction in other forums is permitted, provided the original author(s) and the copyright owner(s) are credited and that the original publication in this journal is cited, in accordance with accepted academic practice. No use, distribution or reproduction is permitted which does not comply with these terms. 